ENSAYOS

\title{
ESTADO DEL ARTE Y FUNDAMENTOS PARA LA CONSTRUCCION DE INDICADORES DE GENERO EN EDUCACION*
}

State of the art and foundations for the gender indicators construction in education

\author{
Estela Arcos G. ${ }^{1}$; Víctor Figueroa A. ${ }^{2}$; Christian Miranda J. ${ }^{3}$, Carmen Ramos ${ }^{4}$ \\ ${ }^{1}$ Instituto Enfermería Materna, Facultad de Medicina, Universidad Austral de Chile. \\ ${ }^{1}$ Casilla 567, Valdivia, Chile, Fono 63-221423, E-mail: earcos@uach.cl \\ ${ }^{2}$ Instituto de Estadística, Facultad de Ciencias Económicas y Administrativas, Universidad Austral de Chile. \\ ${ }^{3}$ Instituto de Filosofía y Estudios Educacionales, Facultad de Filosofía y Humanidades, \\ Universidad Austral de Chile. \\ ${ }^{4}$ Departamento de Economía Aplicada, Facultad de Ciencias Económicas, Universidad de Oviedo, España.
}

\section{Resumen}

En este estudio se ha avanzado en la discusión teórica y operacional del enfoque de género en el ámbito educativo. El documento describe la complejidad teórica y metodológica que en las ciencias sociales se da al momento de elaborar indicadores orientados a informar, trasparentar o evaluar el funcionamiento de los establecimientos educacionales. Ante la ausencia de teorías y modelos generales, se busca investigar en el primer ciclo de la enseñanza media, a partir de la opinión de los actores involucrados, la presencia de algunas variables que permitan diseñar una matriz de indicadores que puedan explicar las relaciones entre todos los factores asociados al género y que influyen en el proceso formativo. Desde esta perspectiva, la búsqueda de indicadores de género en el espacio escolar se constituye en un aporte que el equipo de investigadores pretende entregar al sistema educativo nacional.

Palabras clave: género, indicadores, educación.

\section{Summary}

This study reports the advances in the theoretical and operational discussion of the gender approach in the educational area. The document describes the theoretical and methodological complexity observed in the social sciences when elaborating indicators orientated to reporting, clarifying or evaluating the functioning of the educational establishments. Due to the lack of theories and general models, it is attempted to investigate in the first cycle of the secondary education, from the opinion of the involved actors, the presence of some variables that allow designing an indicator matrix that could explain the relations between all the factors associated to gender that influence the formative process. From this perspective, the search of gender indicators in the school space becomes a contribution that the research team tries to deliver to the educational national system.

Key words: gender, indicators, education.

* $\quad$ Proyecto S-2006-35 Dirección de Investigación y Desarrollo. 


\section{INTRODUCCION}

La democracia participativa eficaz sólo puede ponerse en práctica con la participación equitativa e igualitaria de hombres y mujeres. Cuando la equidad es el objetivo esencial del desarrollo, los derechos económicos, sociales y culturales -que responden a los valores de igualdad, solidaridad, universalidad y no discriminación- incrementan su relevancia y vigencia (Ocampo 2000; Beyer 2000). Para evaluar la igualdad de género en las esferas de participación política y económica el Programa de las Naciones Unidas para el Desarrollo (PNUD) diseñó un indicador sobre discriminación de género (UNICEF 2006). La información oficial muestra que el patrón de desarrollo humano es inequitativo, ante lo cual el organismo internacional instaló la equidad de género como objetivo prioritario en la Agenda del Milenio de las Naciones Unidas, donde sugiere el diseño e implementación de políticas que compensen la desigualdad de trayectorias y no sólo de oportunidades para hombres y mujeres (PNUD 2005; Aguirre et al. 2005).

Para lograr equidad de género hay que eliminar los obstáculos a la igualdad entre los géneros independiente de su origen sociocultural. En este sentido, la educación media es señalada como factor clave para incidir simultáneamente sobre la equidad, el desarrollo integral y el ejercicio de ciudadanía plena (Stacki et al. 2003; Magendzo 2003). En Chile, desde el año 2002 se incluye el enfoque de género como uno de los aspectos estratégicos del Programa de Mejoramiento de la Gestión (PMG), entregando una señal explícita sobre el rol del Estado en la construcción de relaciones más equitativas en políticas, programas y reformas legislativas que consideren las diferencias de género en la distribución de beneficios y tareas sociales. En el PMG se propone una línea estratégica para avanzar en términos de equidad, que consiste en focalizar las acciones en áreas vinculadas a la pobreza y desigualdad, esto es: educación, trabajo, patrimonio, demografía y barreras relacionadas con la discriminación según género, etnia y clase (Rico 1996; Redondo et al. 2005; MINEDUC 2006).

A partir de lo anterior, este estudio asume el desafío de construir una herramienta basada en indicadores de género en el contexto de la educación media en Chile. Esto para conjugar las diferentes dimensiones donde la equidad e igualdad de género puedan ser evaluadas, para posteriormente establecer los mecanismos de validación de la metodología propuesta.

En este documento se presentan los presupuestos conceptuales, teóricos y empíricos para el diseño de indicadores de género. Lo que se busca es, por un lado, contribuir al sistema educacional chileno con indicadores que permitan detectar, agregada y desagregadamente, nudos críticos de equidad e igualdad que tiene el proyecto educativo del primer ciclo de enseñanza media y, por otro, hacer comparaciones y aplicar sistemas de monitoreo frente a intervenciones educativas específicas con perspectiva de género. En términos generales, se asume que la equidad e igualdad de género son producto de un trato imparcial a mujeres y hombres en relación con derechos, beneficios, obligaciones y posibilidades.

\section{MARCO DE REFERENCIA}

I.1. Enfoques de desarrollo, políticas y desigualdades sociales: el contexto. La generación de políticas que pretenden contrarrestar las desigualdades y discriminaciones orientan 
la misión del Estado democrático para crear espacios para la coexistencia de todos sus ciudadanos y ciudadanas (Godoy et al. 2006). Al respecto, la literatura consultada indica que las instituciones, encargadas de la socialización de las políticas, cultura, normas, y valores, habitualmente integran en sus códigos y prácticas formas de reproducción de la discriminación y desigualdad que no siempre son leídas de esta forma (Abramo 2000; Godoy et al. 2006). De esta manera se comprueba que el conocimiento que aportan las investigaciones sobre las desigualdades de género invaden el ambiente sociopolítico, pero no se integran a la argumentación de los grupos con poder de decisión en las transformaciones de la ciudadanía desde una perspectiva de género (Rico 1996; Tarrés 1998; Messina 2001).

Se suma a lo anterior, el hecho de que las prácticas económicas y culturales que afectan el cambio social y equidad de género no cambian rápidamente, porque hay un proceso dialéctico que incluye perspectivas subjetivas y barreras ideológicas sustentadas por normas y sistemas de valores patriarcales que han discriminado y discriminan a las mujeres en la esfera pública y privada (Figueroa 2001; Buvinic 1998; Smulders 2002). El patriarcado se ha concretado en el sistema cultural; se ha perpetuado en el imaginario colectivo por símbolos, ritos y tradiciones y, también, se reproduce en el sistema educativo (Lozada 1999; Stacki et al. 2003; Arcos et al. 2006).

El enfoque de género como categoría de análisis es un instrumento conceptual que ayuda a leer la realidad educativa identificando desigualdades y obstáculos societales que la escuela reproduce. La articulación del género al ámbito escolar significa para las jóvenes asumir posiciones de control en la cultura escolar donde se construyen las relaciones y se distribuye el poder. Significa también para las mujeres participar activamente en los procesos de empoderamiento y cambio educativo promoviendo competencias a fin de procesar formas de relación y maneras de ver el mundo (OCDE 2004).

En síntesis, se asume a partir de la discusión bibliográfica considerar al género como un componente de la educación ciudadana, en tanto es concebido como un elemento constitutivo de las prácticas escolares basados en las diferencias entre los sexos y como forma primaria de relaciones significantes de poder y desarrollo.

I.2. Género y Educación. El concepto de género surge desde los conflictos que provocó el enfoque Mujer y Desarrollo para definir las estrategias orientadas a mejorar la posición social y económica de las mujeres. Se criticó el aislamiento o "ghettizacion" de las mujeres, al sostener que los proyectos se concebían "sólo para mujeres y por mujeres", dando lugar a la marginalización de los estudios de la mujer en las ciencias sociales. La segunda controversia fue la "universalidad" que adopta el concepto de "mujer" porque no representa la diversidad. Al pensar a la mujer como "universal" se alude a una esencia femenina que explica esta universalidad (Najéra et al. 1998; Barbieri 2000).

Producto de estos análisis surgió la necesidad de superar las nociones esencialistas y universalistas y es así como se genera el surgimiento de la categoría "género". Se argumenta que lo que se entiende por ser hombre o ser mujer tiene relación con un aprendizaje sociocultural más que con las características biológicas. El sexo se hereda y el género se adquiere a través de un aprendizaje, de aquí se desprende que, por un lado, la posición de la mujer no está determinada biológicamente sino culturalmente y, por otro, la importancia que tiene el rol de la escuela en dicho proceso de aprendizaje (Cardaci 1998). 
Según las Naciones Unidas (1979), el género constituye una teoría amplia que abarca categorías, hipótesis, interpretaciones y conocimientos relativos al conjunto de fenómenos históricos construidos en torno al sexo. El género como construcción simbólica capta los atributos asignados a las personas a partir de su sexo. Se trata de atributos biosicosociales que influyen sobre la organización de la sociedad en todas sus vertientes y sirven como base para la creación de jerarquías de poder. Para Tarrés (1998), el género se constituye en un complejo mosaico de generación y reparto de poderes que se concretan en maneras de vivir y en oportunidades y restricciones diferenciales.

El género, a partir de su definición proporciona recursos ausentes en otros enfoques, porque asume la complejidad social, cultural y política existente entre mujeres y hombres. Es una visión analítica, explicativa y alternativa que reclama el fin de la intolerancia y la construcción de la mutua aceptación basada en el reconocimiento de la equivalencia humana (Naciones Unidas 1979). Con ello, se propone una visión de la humanidad diversa y democrática que requiere que mujeres y hombres sean distintos a lo que son y eran, para llegar a construir una democracia genérica que reconozca la diversidad.

Entre los fines de la perspectiva de género está la participación en la construcción de una nueva configuración de la historia, la sociedad, la cultura y la política desde las mujeres y con las mujeres (Cardaci 1998). Desde luego, revelar esta configuración de la realidad obliga a plantear nuevos problemas y alternativas y a incluir mujeres en el proceso de desarrollo, la política, el Estado y la vida en democracia.

La estrategia de cambio social de acuerdo con la perspectiva de género empieza con la identificación de diversas cosmovisiones (valóricas, normativas, entre otras) que coexisten en cada sociedad y que se forman preferentemente en la escuela. En esta se procede a detectar las posibilidades y los conflictos que enfrentan los hombres y las mujeres para su desarrollo personal y profesional.

La educación tiene por propósito el desarrollo integral de las personas a fin de ser miembros activos de la sociedad y ejercicio pleno de ciudadanía (Lamas 2000). Para Dorr (1997), la educación es una forma de relación entre personas que permite descubrir, conocer y reconocer la naturaleza, la cultura y las instituciones, para concretar una transformación personal y social que facilite el desarrollo de potencialidades personales y sociales. A través del proceso educativo, la persona construye su identidad simbólicomaterial y expresa su potencial humano, para problematizar y transformar la cultura.

Desde la perspectiva de género se ha identificado la ideología que transmiten los espacios educativos y se ha comprendido cómo se construyen los procesos y discursos en la sala de clases (Cazden 1991). Esta perspectiva ha revelado que los programas educativos no satisfacen necesidades específicas diferenciadas por género $\mathrm{y}$, como consecuencia, el sistema educacional reproduce y refuerza las desigualdades entre los géneros. Se reporta que programas de estudio, métodos de enseñanza, contenidos, textos y materiales educativos privilegian los valores androcéntricos y no cuestionan el rol asignado a mujeres y hombres tanto en el pasado como en el presente (Araneda 1997; Figueroa 2001, Arcos et al. 2006).

En la literatura internacional se evidencia cómo los avances en materia educativa no han tenido su correlato en equidad de género respecto a inserción laboral, niveles de ingreso y participación en los procesos de adopción de decisiones y de poder (Beyer et al. 2002; World Bank 2004; UNICEF 2006). Como consecuencia, los países han debido asumir el desafío de formular una tercera generación de reformas donde se sitúe la 
equidad de género en el centro de las políticas educativas. La preocupación en Chile, respecto a equidad de género en el contexto escolar, se ha centrado en los niveles de aprendizaje de ambos géneros. Un hallazgo importante es que, comparados con las mujeres, los hombres sienten más presión de sus padres para obtener un buen rendimiento y perciben un mayor apoyo para estudiar en el hogar (Andrade y Miranda 2001). Sobre lo anterior, es válido preguntarse si en las familias chilenas la educación de los niños cobra prioridad por sobre la educación de las niñas y, en el caso de la educación si el hecho de ser mujer influye en las percepciones de éxito o fracaso que los docentes tienen sobre el futuro de sus estudiantes. De ser ciertas, tales diferencias constituyen desafíos para el principio de equidad que guía la reforma educacional (Richardson 2003; MINEDUC 2005).

Sobre lo antes mencionado, se ha reportado que en el nivel secundario se acentúa la desigualdad basada en el género. Al respecto, el estudio MIDEPLAN (2003) muestra cómo una de cada cuatro niñas chilenas que abandonan la educación media antes de concluir sus estudios lo hace en una etapa más temprana que los niños, sobre todo en zonas de alta vulnerabilidad sociocultural. El matrimonio precoz, el embarazo en la adolescencia y la necesidad de prestar asistencia a la familia son algunas de las causas de la deserción escolar entre las niñas (UNICEF 2006).

Para minimizar el efecto "mujer" se han implementado políticas educativas orientadas a fortalecer el posicionamiento académico y rol económico de las jóvenes en la educación terciaria y sector productivo. Se ha reportado que hijos e hijas de madres instruidas viven más tiempo y gozan de mejor salud, pues resulta mucho más probable que estas mujeres soliciten oportunamente atención de salud profesional. Además, se ha confirmado que se fortalecen en ella y su descendencia capacidades y competencias tales como el pensamiento crítico, la resolución de problemas, emprendimiento, entre otras (Kuninobu 2003).

El enfoque de género reconoce en el espacio educativo cuatro tipos de currículo: el discurso pedagógico, Formal, y, en la práctica pedagógica, En acción, Obviado y Oculto. El currículo Formal hace referencia al enfoque pedagógico de la metodología, a los recursos, contenidos, criterios de evaluación, etc. En el segundo, a las emergencias que se generan en el espacio escolar, tales como acontecimientos históricos, sociales o de la cultura que rodea a la Institución. En el Oculto se refleja lo que sucede en la ejecución de los cursos, en el aula o el taller, es decir, lo que no está previsto formalmente, respecto a las interacciones entre docentes y estudiantes tales como: formas de comunicación, mensajes subliminales respecto a la condición femenina o masculina, uso diferenciado de los espacios, entre otras. El currículo oculto juega un papel importante en la configuración de significados y valores, la mayoría de las veces no es consciente por lo que es difícil reconocerlo. Finalmente, el Obviado hace referencia a un conjunto de situaciones que se presentan en los procesos de enseñanza-aprendizaje. Son los temas no tratados, el ignorar interrogantes respecto a temas transversales, ignorar sentimientos, necesidades, intereses, experiencias y conocimientos (Dorr 1997; OIT-UN 1998; CINTERFOR-OIT 2003).

Respecto a lo que se produce en los liceos, se ha señalado que la igualdad de género en la profesión docente es importante por cuanto condiciona los resultados del proceso de enseñanza-aprendizaje, aunque todavía no se ha podido establecer científicamente que exista una relación directa entre la equidad de género y los resultados escolares. 
No obstante, se asume que la igualdad de género incide en el funcionamiento interno de un establecimiento, en el que suele predominar el personal femenino, por lo menos en lo que se refiere a la igualdad de oportunidades y de trato, al grado de satisfacción o insatisfacción en el trabajo y a la forma en que se organiza la enseñanza (Colegio de Profesores 1997). Sin duda, faltan indicadores que permitan fundamentar tales apreciaciones a fin de optimizar la calidad de la educación.

I.3. Modelos e indicadores de género en educación. Para introducir el enfoque de género en los procesos de formación de la educación secundaria es necesario analizar los procedimientos y considerar los indicadores en cada una de las fases del desarrollo curricular (Vianna 2004). Más aún si se quiere intervenir en el primer ciclo, donde se presentan las más altas tasas de deserción (MIDEPLAN 2003), se requiere diseñar indicadores de género que permitan diagnosticar (transversal y longitudinalmente) y monitorear equidad e igualdad, en un antes, un durante y un después, desde lo general (políticas, programas de estudio) a lo particular (dirección, docencia, planificaciones de aula, etc.) (Dávila 2004).

De acuerdo a la CEPAL (1999), el objetivo de definir indicadores de género es imprescindible para monitorear el cumplimiento de los acuerdos llevados a cabo en el ámbito internacional y hacer un seguimiento de la situación de mujeres y hombres, según los documentos que refrendan los aspectos acordados. Las mismas clasificaciones planteadas para los indicadores en general son válidas para los indicadores de género, así por ejemplo, en el caso de los indicadores de carácter económico, la eficacia pasa por estrechar brechas de género, por lo cual se busca medir la situación diferencial entre hombres y mujeres en un inicio y al final del programa. Por su parte los indicadores de eficiencia buscan incorporar en el análisis los recursos empleados por mujeres y hombres en los fondos públicos destinados a la igualdad y los resultados del programa sobre éstos $\mathrm{y}$, finalmente, los indicadores de impacto que tratan de efectuar análisis desagregados según sexo, tanto para las personas afectadas (efectos directos), como para el colectivo (beneficiarios indirectos).

En el caso de la clasificación según la naturaleza del objeto a medir, se potencian indicadores de realización desagregados por sexo, esto significa especificar, por ejemplo, los recursos (hombres y/o mujeres contratadas para realizar el programa) y el uso que se ha dado a los recursos. Por su parte, los indicadores de resultado permiten obtener una fotografía de los logros para mujeres y para hombres, y los de impacto visualizar los impactos previstos e imprevistos, así como también apreciar los efectos diferenciales para mujeres y hombres.

En el caso de la educación, la exigencia de mayor calidad está estrechamente relacionada con el desarrollo de indicadores de género para analizar su funcionamiento. No es extraño, por ello, que en los últimos años se hayan generado modelos orientados a promover su utilización. A este interés académico ha contribuido también la creciente presión social y estudiantil por mejorar el funcionamiento y la calidad del servicio que el Estado o los privados se comprometen a entregar. Hay una exigencia de equidad, transparencia e información en la misma medida que hay un mayor compromiso y confianza en los beneficios que la educación produce en la sociedad y en las personas.

En la literatura consultada, tal como en el caso de la definición de indicador, los modelos que buscan operacionalizar el constructo están lejos de entregar una respuesta 
robusta en términos científicos. Tal como señala Marchesi y Martí (2000), el paso de un concepto a un instrumento de medida es una larga y pesada tarea, ya que un concepto puede generar decenas de indicadores.

En educación se ha pasado desde el modelo input-output a enfoques más amplios, basados en variables estructurales (contexto, proceso) y de resultado (efectos). Los modelos más recientes, a partir de perspectivas organizacionales, otorgan mayor peso a indicadores relacionados a la toma de decisiones, opinión de los actores y teorías, ampliando con ello el concepto de calidad educativa al ámbito social o cohesión moral de sus miembros.

En el ámbito internacional, se destaca el Proyecto Internacional de Indicadores de la Educación (INES) impulsado por la OCDE desde la década de los 80. A través de una actualización periódica tal organización ha definido tres factores determinantes: Costes, recursos y procesos escolares; contexto, y resultados. El lugar que ocupa el género en este modelo está dado por constituirse en una variable del factor contexto junto a nivel de formación de la población (OCDE 1995).

Respecto a los criterios para definir indicadores de género se recomienda conjugar dos criterios: i) un criterio sistémico que contribuya a abordar diferenciadamente los distintos subsistemas, para potenciar allí la mayor igualdad de oportunidades y, ii) un criterio sinérgico, según el cual la mayor igualdad de oportunidades en un campo puede impactar favorablemente la igualdad de oportunidades en otros. Dentro de las principales características destacan la validez, utilidad, viabilidad, comparabilidad, comprensión, independencia, oportunidad, posibilidad de agrupación y desagregación. Para la selección de indicadores de género se sugiere incorporar indicadores cualitativos y cuantitativos que puedan ser usados para una validación cruzada de cada uno, en un proceso llamado triangulación Por regla general, se recomienda sobre seis indicadores por cada etapa, considerando la factibilidad de uso, costo y tiempo comprometido en la recolección y análisis de comprensivo de resultados (CIDA 1997).

En síntesis, en este estudio se ha avanzado en la discusión teórica y operacional del enfoque de género en el ámbito educativo. El documento describe la complejidad teórica y metodológica que en las ciencias sociales se da, al momento de elaborar indicadores orientados a informar, trasparentar o evaluar el funcionamiento de los establecimientos educacionales. Ante la ausencia de teorías y modelos generales, se busca investigar en el primer ciclo de la enseñanza media, a partir de la opinión de los actores involucrados, la presencia de algunas variables que permitan diseñar una matriz de indicadores que puedan explicar las relaciones entre todos los factores asociados al género y que influyen en el proceso formativo. Desde esta perspectiva, la búsqueda de indicadores de género en el espacio escolar se constituye en un aporte que el equipo de investigadores pretende entregar al sistema educativo nacional.

\section{BIBLIOGRAFÍA}

Abramo, L., M. E. Valenzuela, M. Pollack (2000). Equidad de género en el mundo del trabajo en América Latina. Avances y desafíos cinco años después de Beijing. Documento 130, Oficina Internacional del Trabajo, Oficina regional para América Latina y el Caribe, $83 \mathrm{pp}$.

Aguirre, R., C. García, C. Carrasco (2005). El tiempo, los tiempos, una vara de desigualdad. Serie 65 Mujer y Desarrollo CEPAL. Disponible en: http://www.eclac.cl/cgi-bin/getProd.asp?xml=/publicaciones/xml/7/22367/P22367.xml\&xsl=/mujer/tpl/p9f.xsl\&base=/mujer/tpl/top-bottom.xsl 
Andrade, M., Ch. Miranda (2001). Predicción del rendimiento académico lingüístico y lógico matemático por medio de las variables modificables de las inteligencias múltiples y del hogar. Boletín de Investigación Educacional 16: 301-315. Santiago: Pontificia Universidad Católica de Chile.

Arcos, E., I. Molina, E. Fecci et al. (2006). Descubriendo el género en el currículo explícito (currículo formal) de la educación de tercer ciclo, Universidad Austral de Chile 2003-2004. Estudios Pedagógicos 32:33-47.

Arcos, E., I. Molina, R. E. Trumper, L. Larrañaga, P. Smulewicz, M. I. del Río, M. Uarac (2006). Estudio de perspectiva de género en estudiantes y docentes de la Universidad Austral de Chile. Estudios Pedagógicos 32:27-45.

Araneda, P., J. Guerra, M. Rodríguez (1997). Lo femenino visible. Publicado por Ministerio de Educación, Corporación de Promoción Universitaria, Servicio Nacional de la Mujer, Santiago de Chile.

Barbieri, T. (2000). Sobre la categoría de género: una introducción teórico metodológica. En: Montecino, S. y Donoso, C. Teorías de género, Modulo I, Centro Interdisciplinario de Estudios de Género, Facultad de Ciencias Sociales, Universidad de Chile, 87-104.

Beyer, H. (2000). Educación y desigualdad de ingresos: una nueva mirada. Estudios Públicos 77 Diasponible en: http://www.cepchile.cl/dms/lang_1/doc_1418.html. Consultado en septiembre 2006

Beyer, H y C. LE Foun (2002). Un recorrido por las desigualdades salariales en Chile. Estudios Públicos 85. Disponible en: http://www.cepchile.cl/dms/lang_1/doc_3031.html

Buvinic, M. (1998). Mujeres en la pobreza: Un problema global. Banco Interamericano de Desarrollo. Disponible en: http://www.iadb.org/sds/doc/769spa.pdf

CIDA (1997). Guide to gender sensitive indicators. Canadian International Development Agency. Disponible en: http://w3.acdi-cida.gc.ca

Cardaci, D. (1998). Nuevos entramados: familia, salud y organizaciones de mujeres. En: Figueroa, P. La condición de la mujer en el espacio de la salud. Ed por El Colegio de México, 243-265.

Cazden, C. (1991). El discurso en el aula. Barcelona: Paidós.

CEPAL (1999). Indicadores de género para el seguimiento y la evaluación del Programa de Acción Regional para las Mujeres de América Latina y el Caribe, 1995-2001, y la Plataforma de Acción de Beijing. Santiago, Chile: Publicaciones CEPAL.

Cinterfor-OIT. (2003). Género y formación por competencias: aportes conceptuales, herramientas y aplicaciones. Programa Formujer. CINTERFOR-OIT. Disponible en: http://www.ilo.org/ public/spanish/region/ampro/cinterfor/publ/com_gen/index.htm

Colegio de Profesores, A.G. (1997). Informe Final. Primer Congreso Nacional de Educación. Santiago: sin editorial.

Dávila, M. (2004). Indicadores de género. Universidad de Oviedo, 2004.

Dorr, A., Sierra, G. (1997). El currículo oculto de género. Infancia, Solidaridad en Internet Disponible en http://www.risolidaria.tdata.cl/Portada/Dirseccion/Home_Infancia.asp?dir=Documentos_de_ Analisis_IF\&id=4168

Figueroa, J. G. (2001). Los procesos educativos como recurso para cuestionar modelos hegemónicos masculinos Diálogo y Debate de Cultura Política, Centro de Estudios para la Reforma del Estado, 4(15-16):7-32.

Godoy, P., Solís, D. (2006). Una mirada exploratoria de las necesidades en educación de la sexualidad, afectividad y género de niños, niñas y jóvenes con discapacidad intelectual. Secretaría Técnica de Educación en Sexualidad, Afectividad y Género, Unidad de Educación Especial, División de Educación General, Ministerio de Educación de Chile. Disponible en: http://www. mineduc.cl/biblio/documento/200612221240260.Informe\%20final\%20ed\%20sexual\%20y\%20 discapacidad\%20intelectual.pdf 
Kuninobu, J. (2003). Cooperación internacional, educación de la mujer y justicia de género. Instituto de Cooperación Internacional de la Asociación Alemana para Educación de Adultos. Disponible en: http://www.iiz-dvv.de/spanisch/default.htm

Lamas, M. (2000). La antropología feminista y la categoría de género. En: Montecino, S. y Donoso, C. Teorías de género, Módulo I. Centro Interdisciplinario de Estudios de Género, Facultad de Ciencias Sociales, Universidad de Chile, 57-84

Lozada, B. (1999). Una constelación Foucaultiana de la femineidad. Poder y Género. Resumen de la Tesis de Maestría presentada al CIDES - UMSA. Umbrales, 5. CIDES, Postgrado en Ciencias del Desarrollo, UMSA, Universidad Mayor de San Andrés, La Paz, Bolivia. Disponible en: http://bibliotecavirtual.clacso.org.ar/ar/libros/bolivia/cides/umbrales/umbrales5.pdf

Magendzo, A. (2003). Formación ciudadana y objetivos transversales. En Hevia, R. (editor). La educación en Chile hoy. Cáp. V (335-346). Santiago: Universidad Diego Portales.

Marchesi, A., E. Martí (2000). Calidad de la enseñanza en tiempos de cambio. Madrid: Alianza.

Messina, G. (2001). Estado del arte de la igualdad de género en la educación básica de América Latina (1990-2000). UNESCO. Disponible en: http://www.unesco.cl/medios/biblioteca/documentos/estado_arte_igualdad_genero_ed_basica_lac_esp.pdf

Ministerio de Educación, Chile. (2005). Análisis de las diferencias de logro en el aprendizaje escolar entre hombres y mujeres. Sistema de Medición de la Calidad de la Educación, SIMCE. Disponible en: www.mineduc.cl/biblio/documento/200612261203330.informegenerosimce.pdf

Ministerio de Educación, Chile. (2006). Aplicación del enfoque de género a las actividades del MINEDUC. Ministerio de Educación, División de Planificación y Presupuesto, Departamentos de Estudios y Desarrollo. Disponible en: www.mineduc.cl/biblio/documento/200612270933050. guia\%20etapa_iv\%20pmg\%20genero\%202006.pdf

MIDEPLAN. (2003). Encuesta de Caracterización Socioeconómica. Santiago. Disponible en: http:// www.mideplan.cl/casen/pdf/desercionescolar.pdf

Naciones Unidas. (1979). Convención sobre la eliminación de todas las formas de discriminación contra la mujer, CEDAW, División para el Avance de la Mujer http://www.un.org/womenwatch/daw/cedaw

Naciones Unidas, CEPAL. (2000). El desafío de la equidad de género y de los derechos humanos en los albores del siglo XXI. Octava conferencia sobre la Mujer de América Latina y el Caribe, CEPAL: 75 pp.

Naciones Unidas, PNUD. (2001). Informe sobre desarrollo humano 2001: Poner el adelanto tecnológico al servicio del desarrollo humano. Disponible en: <http://www.undp.org/hdr2001/ spanish/spcptodo.pdf>

Naciones Unidas, PNUD. (2002). Informe sobre desarrollo humano 2002. Disponible en: http:// www.undp.org/hdr2002/espanol/espanolPR.pdf

Ocampo, J.A. (2000). En: equidad, desarrollo y ciudadanía: Parte I. La visión global. Publicaciones CEPAL, Santiago, Chile. Disponible en: http://www.eclac.cl/publicaciones/xml/5/4425/ lcg2071.pdf

OCDE (1995). Education at a Glance/Regards sur l'education. París: OCDE.

OCDE (2004). Informe del Comité Técnico sobre el sistema educativo chileno. Santiago: MINEDUC.

OIT-NU. (1998). La educación permanente en el siglo XXI: nuevas funciones para el personal de educación. Disponible en: http://www.ilo.org/public/spanish/dialogue/sector/techmeet/ jmep2000/jmepr1.htm

Naciones Unidas PNUD. (2005). Informe sobre desarrollo humano. México 2004. Programa de las Naciones Unidas para el Desarrollo. Disponible en: http://saul.nueve.com.mx/informes/ index.hyml

Naciones Unidas PNUD. (2006). Una alianza mundial para el desarrollo. Programa de las Naciones Unidas para el Desarrollo. Informe anual 2006. Disponible en: http://www.undp.org/spanish/ publicaciones/informeanual2006/iar06_sp.pdf 
Redondo, J., C. Descouvieres, K. Rojas (2005). Eficacia y eficiencia en la enseñanza media chilena desde los datos simce 1994, 1998 y 2001. Revista Enfoques Educacionales 7:125-144.

Richardson, J. \& A. Woodley (2003). Another look at the role of age, gender and subject as predictors as academic attainment in higher education. Studies in Higher Education 28:475-493.

Rico, N. (1996). Formación de los recursos humanos femeninos: Prioridad del crecimiento y de la equidad. Serie Mujer y Desarrollo 15, CEPAL. Disponible en: http://www.eclac.org/cgibin/getProd.asp?xml=/publicaciones/xml/6/5686/P5686.xml\&xsl=/mujer/tpl/p9f.xsl\&base=/ mujer/tpl/top-bottom.xsl

Smulders, A. (2002). Género e identidad en el contexto del debate sobre la modernidad. En publicación: Umbrales, $\mathrm{N}^{\circ}$ 11. CIDES, Postgrado en Ciencias del Desarrollo, UMSA, Universidad Mayor de San Andrés, La Paz, Bolivia. Disponible en: http://bibliotecavirtual.clacso.org.ar/ ar/libros/bolivia/cides/umbrales/umbrales11.pdf

Stacki, S. \& K. Monkman (2003). Change Through Empowerment Processes: women's stories from South Asia and Latin America. Compare, 33:173-189.

Tarrés, M. L. (1998). ¿Importa el género en la política? En Género y cultura en América Latina, Cultura y participación política. Ed Colegio de México, pp. 13-32.

UNICEF (2006). (Estado Mundial de la infancia 2007, UNICEF. Disponible en: http://www.unicef. org/spanish/sowc07/docs/sowc07_sp.pdf

Vianna, C. (2005). La inclusión del género en las políticas públicas educativas en Brasil: Cambios curriculares versus capacitaciones puntuales. Seminario Internacional Equidad de Género en las Reformas Educativas de América Latina. Disponible en: http://www.hexagrama.cl/seminario/ ponencias/claudiavianna.pdf (Consultado en diciembre 2006).

World Bank (2004). Promote gender equality and empower women. The World Bank Group, Global Data Monitoring Information System. Disponible en: http://ddp-ext.worldbank.org/ ext/GMIS/gdmis.do?siteId=2\&goalId=7\&menuId=LNAV01GOA3 